\title{
Correlation between Stromal Cell-Derived Factor 1 and the Prognosis of Transient Ischemic Attack
}

\author{
Mingjie Liu ${ }^{1}$, Jing Xie ${ }^{2,}$, Xinxin Zheng ${ }^{1}$, Mengmeng Wang ${ }^{1}$, Guanglong Chen ${ }^{1}$, Tao Wang ${ }^{1}$ and $\mathrm{Yi} \mathrm{Lu}^{2}$ \\ ${ }^{1}$ Department of clinical medicine, Bengbu Medical College, Anhui, Bengbu, People's Republic of China \\ 2 Department of Neurology, the First Affiliated Hospital of Bengbu Medical College, Anhui, Bengbu, People's Republic of China
}

* Corresponding author: Jing Xie, Department of Neurology, the First Affiliated Hospital of Bengbu Medical College, Anhui, Bengbu, People's Republic of China. Tel: +8618895669639; Email: 23899224@qq.com

Received 2021 February 09; Revised 2021 February 28; Accepted 2021 April 16.

\begin{abstract}
Background: Transient Ischemic Attack (TIA) (1) is a neurological dysfunction of transient cerebrovascular ischemia, which is more common in clinical practice. The risk of further progression to ischemic stroke after TIA can be used as a strong early warning signal of cerebral infarction.

Objectives: The present study aimed to explore the correlation between stromal cell-derived factor 1 (SDF-1) and the prognosis of TIA. Methods: A number of 65 patients with TIA were collected, the ABCD2 clinical risk prediction score was implemented, relevant tests and nuclear magnetic resonance imaging (MRI) were performed, and the SDF-1 was recorded in serum levels. End-point events were selected in patients after cerebral infarction in the short term. The statistical analysis method was used to evaluate TIA short-term development for the occurrence of cerebral infarction after risk, the severity of serum level of SDF-1, and infarction.

Results: Based on the results, the high-risk group, middle-risk group, and low-risk group had statistically significant differences in serum SDF-1 levels $(\mathrm{F}=3.820 ; \mathrm{P}<0.05)$. Correlation analysis demonstrated that $\mathrm{ABCD} 2$ score was positively correlated with serum SDF-1 $(\mathrm{r}=0.349 ; \mathrm{P}<0.05)$. End-point events were included in the occurrence group and not included in the non-occurrence group. The SDF-1 level of the occurrence group was significantly higher than that of the non-occurrence group. Based on the cranial MRI results as the gold standard, the areas under the curve of the receiver operating characteristic curve (ROC) drawn based on the SDF-1, ABCD2 score, and SDF-1 combined with the ABCD2 score on the end-point events of TIA patients were obtained at $0.717,0.697$, and 0.762 , respectively. The sensitivity and specificity of SDF-1 were reported as $77.8 \%$ and $68.1 \%$, respectively. The sensitivity and specificity of the ABCD2 score were $83.3 \%$ and $48.9 \%$, respectively. The sensitivity and specificity of SDF-1 combined with the ABCD2 score were $72.2 \%$ and $76.6 \%$, respectively.

Conclusion: As evidenced by the obtained results, SDF-1 is associated with ABCD2 score risk classification. Patients with high levels of SDF-1 combined with the ABCD2 score have a higher risk of cerebral infarction. Elevated SDF-1 levels may indicate that TIA patients have a poor short-term prognosis and have a certain predictive value for the diagnosis of the risk of ischemic stroke in the short term.

Keywords: Cerebral infarction, Chemokine CXCL12, Transient ischemic attack
\end{abstract}

\section{Background}

Transient Ischemic Attack (TIA) (1) is a neurological dysfunction of transient cerebrovascular ischemia, which is more common in clinical practice. The risk of further progression to ischemic stroke after TIA can be used as a strong early warning signal of cerebral infarction. Studies have demonstrated that about $1 / 3$ of TIA patients with frequent short-term attacks can have a secondary stroke (2). The ABCD2 clinical risk prediction scale (3) is the main scoring tool for assessing the risk stratification of TIA. Nonetheless, in recent years, some scholars have questioned the accuracy of the ABCD 2 score in distinguishing between high-risk and low-risk TIA patients. The clinical value of predicting secondary cerebral infarction in TIA patients can be improved by a combination of ABCD2 score and imaging examination based on the original score (4). Nevertheless, primary hospitals in China have limited medical resources, restricting the access of some urban and rural residents due to high costs of testing; therefore, it is not practical to promote the new scoring method.

\section{Objectives}

The current study aimed to find a rapid and effective biomarker plasma chemokine, stromal cellderived factor 1 (SDF-1) (5), to explore the predictive value of its levels in the risk of short-term (within 7 days) ischemic stroke after TIA and cerebral infarction, as well as its diagnostic role.

\section{Methods}

\subsection{Study Population}

A total of 65 patients diagnosed with TIA in the First Affiliated Hospital of Bengbu Medical College were enrolled in the present study. In terms of gender, the majority of subjects $(n=39)$ were male. The length of hospitalization was all> 7 days. All the patients met the diagnostic criteria adopted by the Fourth Congress of Chinese Cerebrovascular Diseases (6), and all signed informed consent prior to participation in the study.

\subsection{Data collection}

The venous blood of the selected patients was

Copyright (c) 2021, Author(s). This is an open-access article distributed under the terms of the Creative Commons Attribution-NonCommercial 4.0 International License (http://creativecommons.org/licenses/by-nc/4.0/) which permits copy and redistribute the material just in noncommercial usages, provided the original work is properly cited 
collected within $24 \mathrm{~h}$ to detect blood routine, liver and kidney functions, ions, blood lipids, blood glucose, glycated hemoglobin, coagulation function, SDF-1, and other indicators. Immediately after the examination and inspection, all patients were scored with ABCD2. Based on the obtained score, the patients were assigned to three groups, including low-risk group (0-3 points), medium risk group (4-5 points), and high-risk group (6-7 points). The patients with TIA within 7 days of cerebral infarction were used as the end-point event, the non-occurred end-point event was categorized in the non-occurring group, and the occurred end-point event was categorized in the occurring group.

\subsection{Statistical analysis}

Data were analyzed in SPSS software (version 25), the measurement data was expressed as $(\bar{\chi} \pm \mathrm{s})$, and the comparison between the two groups was made by independent sample t-test or F-test. The count data was expressed as a percentage (n\%), and the chi-square test was utilized for comparing the two groups. The correlation analysis between serum SDF1 level and ABCD2 score in TIA patients was performed using Pearson correlation analysis. The receiver operating characteristic (ROC) curve was used to determine the serum SDF-1 level, ABCD 2 score, SDF-1 level combined with ABCD 2 score, and other multiple indicators to judge the prognosis of TIA patients. A p-value less than 0.05 was considered statistically significant.

\section{Results}

4.1 Comparison of general data of transient ischemic attack patients with different prognosis

End-point events were included in the occurrence group, while they were not considered in the nonoccurrence group. As displayed in Table 1, the serum SDF-1 level in the occurrence group was significantly higher than that in the non-occurrence group, and the difference was statistically significant $(\mathrm{t}=2.794$; $\mathrm{P}=0.007$ ). The mean age scores of two groups of occurrence and non-occurrence were reported as $62.61 \pm 16.17$ and $61.96 \pm 15.33$ years, respectively. There was no significant difference between the two groups $(\mathrm{P}=0.880)$. Moreover, the gender composition ratio between the two groups was not statistically significant $(\mathrm{P}=0.910)$.

\subsection{Comparison of serum SDF-1 levels in patients with} different degrees of illness and the correlation between SDF-1 and ABCD2 scores

As illustrated in Table 2, 26, 30, and 9 patients with TIA were included in the low-risk, medium-risk group, and high-risk groups, respectively. The three groups had statistically significant differences in serum SDF-1 levels ( $\mathrm{F}=3.820 ; \mathrm{P}=0.027)$. The results of further multiple comparisons indicated that the difference between the high-risk group and the lowrisk group $(\mathrm{P}=0.009)$ and the medium-risk group $(\mathrm{P}=0.02)$ reached a significant level. Nonetheless, the difference between the medium-risk and low-risk groups was not statistically significant $(\mathrm{P}=0.63)$. Correlation analysis showed that ABCD2 score was positively correlated with serum SDF-1 ( $\mathrm{r}=0.349$; $\mathrm{P}=0.004$ ) (Figure 1).

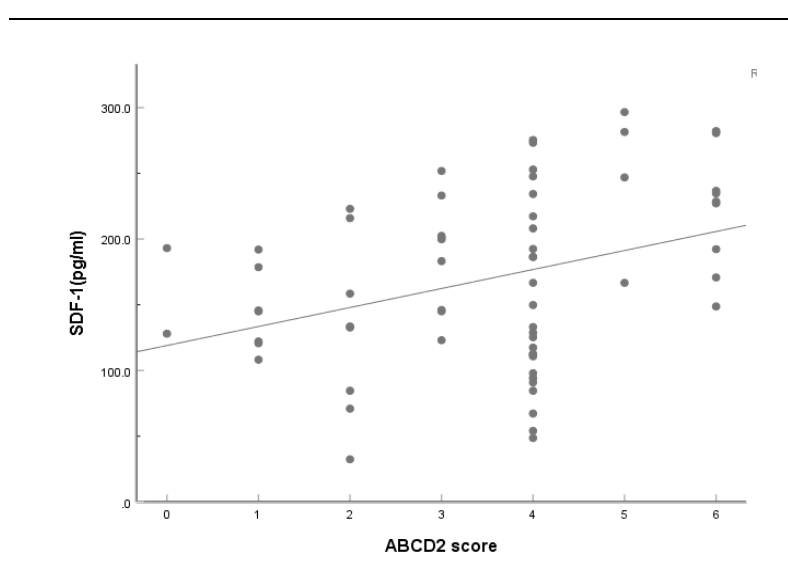

Figure 1. Correlation between SDF-1 level and ABCD2 score in transient ischemic attack patients ABCD2 score was positively correlated with serum SDF-1 $(\mathrm{r}=0.349 ; \mathrm{P}=0.004)$

\begin{tabular}{|c|c|c|c|c|}
\hline index & Non-occurring group & Occurrence group & $\mathbf{t} / \chi^{2}$ & $\mathbf{P}$ \\
\hline Male / Female (n) & $28 / 19$ & $11 / 7$ & 0.013 & 0.910 \\
\hline age & $61.96 \pm 15.33$ & $62.61 \pm 16.17$ & 0.152 & 0.880 \\
\hline SDF-1 $\mathrm{pg} / \mathrm{mL})$ & $156.38 \pm 63.41$ & $204.58 \pm 58.95$ & 2.794 & 0.007 \\
\hline \multicolumn{5}{|c|}{ Table 2. Comparison of SDF-1 levels of transient ischemic attack patients with different disease degrees $(\bar{x} \pm s)$} \\
\hline & Number of cases & \multicolumn{3}{|c|}{ Serum SDF-1 $\quad$ pg/mL) } \\
\hline Low-risk group & 26 & \multicolumn{3}{|c|}{$156.57 \pm 52.49$} \\
\hline Middle-risk group & 30 & \multicolumn{3}{|c|}{$165.34 \pm 74.03$} \\
\hline High-risk group & 9 & \multicolumn{3}{|c|}{$222.42 \pm 45.25$} \\
\hline $\mathbf{F}$ & & \multicolumn{3}{|c|}{3.820} \\
\hline $\mathbf{P}$ & & \multicolumn{3}{|c|}{0.027} \\
\hline
\end{tabular}




\begin{tabular}{|c|c|c|c|c|c|c|c|c|}
\hline \multicolumn{2}{|c|}{ Serum SDF-1 (pg / mL) } & & \multicolumn{2}{|c|}{ Non-occurring group } & Occurrence group & total & \multicolumn{2}{|c|}{ Incidence n (\%) } \\
\hline \multicolumn{2}{|c|}{ Group A $(<100)$} & & \multicolumn{2}{|c|}{9} & 1 & 10 & \multicolumn{2}{|c|}{10.00} \\
\hline \multicolumn{2}{|l|}{ Group B (100-200) } & & \multicolumn{2}{|c|}{25} & 8 & 33 & \multicolumn{2}{|c|}{24.24} \\
\hline \multicolumn{2}{|l|}{ Group C $(>200)$} & & \multicolumn{2}{|c|}{13} & 9 & 22 & \multicolumn{2}{|c|}{40.91} \\
\hline \multirow{2}{*}{ variable } & \multirow{2}{*}{ AUC } & \multirow{2}{*}{$\begin{array}{c}\text { Standard } \\
\text { error }\end{array}$} & \multirow{2}{*}{$P$ value } & \multicolumn{2}{|c|}{ Progressive $95 \%$ confidence interval } & \multirow{2}{*}{ Specificity } & \multirow{2}{*}{ Sensitivity } & \multirow{2}{*}{ Youden Index } \\
\hline & & & & Lower limit & Upper limit & & & \\
\hline SDF-1 & 0.717 & 0.071 & 0.007 & 0.578 & 0.856 & $68.1 \%$ & $77.8 \%$ & 0.459 \\
\hline ABCD2 & 0.697 & 0.072 & 0.015 & 0.555 & 0.838 & $48.9 \%$ & $83.3 \%$ & 0.322 \\
\hline Combined factor & 0.762 & 0.062 & 0.001 & 0.641 & 0.882 & $76.6 \%$ & $72.2 \%$ & 0.488 \\
\hline
\end{tabular}

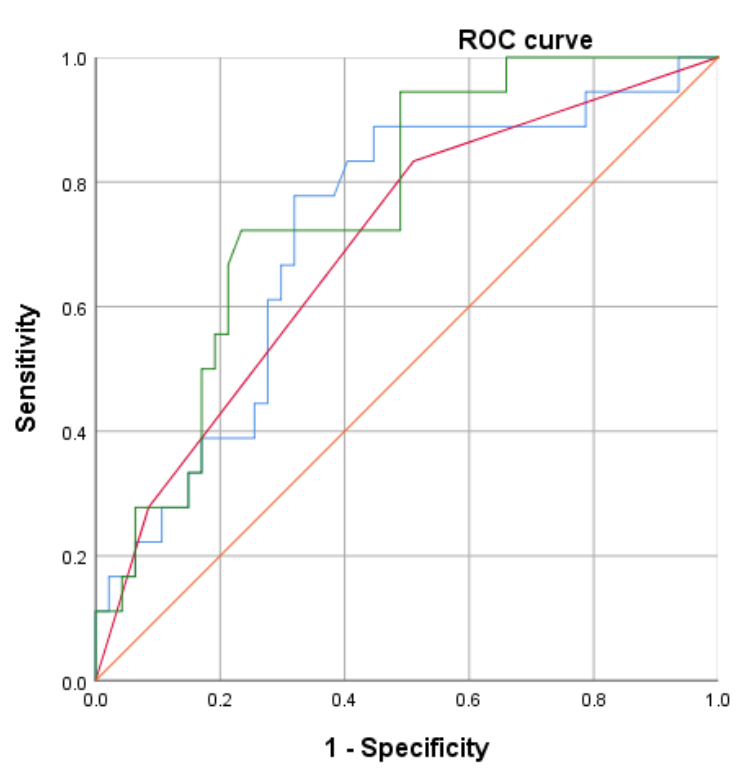

\section{Curve source}

- SDF-1(pg/ml)

- $\mathrm{ABCD2}$ score (splitted)

- Combined factor

- Reference line

Diagnoal segmenents are produced by ties

Figure 2. ROC curve of the predictive value of SDF-1, ABCD2, and combined factors on the end-point event of transient ischemic attack patients.

4.3. Analysis of serum SDF-1 level and incidence of endpoint events

As depicted in Table 3, based on serum SDF-1 levels, patients were assigned to three groups: Group A ( $\mathrm{n}=10,<100 \mathrm{pg} / \mathrm{mL})$, Group B ( $\mathrm{n}=33,100-200 \mathrm{pg} /$ $\mathrm{mL})$ and Group C $(\mathrm{n}=22,>200 \mathrm{pg} / \mathrm{mL})$. The incidence of end-point events exhibited an increasing trend with the elevation of serum SDF-1 levels among patients in different groups. The comparison among groups $\mathrm{A}, \mathrm{B}$, and $\mathrm{C}$ displayed statistically significant differences $(\mathrm{P}<0.05)$.

4.4. The predictive value of multiple indicators such as SDF-1, ABCD2 score, SDF-1 combined with ABCD-2 score for end-point events in patients with transient ischemic attack

As illustrated in Table 4, the areas under the curve of the receiver operating characteristic curve (ROC) drawn based on the SDF-1, ABCD2 score, and SDF-1 combined with the ABCD2 score on the end-point events of TIA patients were obtained at $0.717,0.697$, and 0.762 , respectively. The sensitivity and specificity of SDF-1 were reported as $77.8 \%$ and $68.1 \%$, respectively. The sensitivity and specificity of the ABCD2 score were $83.3 \%$ and $48.9 \%$, respectively. The sensitivity and specificity of SDF-1 combined with the ABCD2 score were $72.2 \%$ and $76.6 \%$, respectively. Figure 2 displays the ROC curve of the predictive value of multiple indicators, such as SDF-1, ABCD2 score classification, and combination factors, on the end-point events of TIA patients.

\section{Discussion}

Prevention of acute ischemic stroke is the key to the wellbeing of patients. Since the 20th century, the prognosis of TIA has evolved with time and has been refined after extensive clinical trials. At present, the ABCD2 scale is primarily used; however, due to its limitations, some imaging and etiology elements have been added to increase its predictive value.

The TIA for short-term recurrent seizures is a 
strong warning signal of acute cerebral infarction. The accurate assessment of early-onset, as well as timely and effective interventions, can improve the prognosis and reduce the risk of secondary cerebral infarction, which in turn, can prevent acute ischemic stroke. The increasing attention to TIA prognosis has led to the emergence of various evaluation scales which are gradually improving in clinical trials. ABCD2 scoring is the primary method of current assessment; nonetheless, due to its limitations, many scholars have begun to introduce etiology and imaging data to increase the predictive value of this scale $(7,8)$. Current barriers to the treatment of cerebral infarction prompted us to look for better prevention methods. The main pathological features of ischemic cerebrovascular disease are neuronal damage and death caused by ischemia and hypoxia, which in turn, result in damages to nervous system function. Ingram (9) et al. conducted a study on the enhancement of the expression of erythropoietin genes under hypoxic conditions. They found that Hypoxia-inducible factor 1-alpha (HIF-1 $\alpha$ ) is a key factor in protecting against hypoxic-ischemic brain injury. The HIF- $1 \alpha$ induces the production of a large number of targeted cells, enhancing SDF-1 expression by targeted gene regulation SDF-1 transcription process. The HIF- $1 \alpha$ which is induced by ischemic damage to the brain is heavily activated in brain cells to achieve the production of blood vessels under the induction of ischemic damage to the brain (10).

The chemokine factor is a class of cytokines that have a tendency effect on different cells. They participate in blood vessel production, Atherosclerosis, inflammatory response in tumors, as well as AIDS and other physiological or pathological processes $(11,12)$. The SDF-1 as a member of the chemokine factor family binds to the receptor CXCR4 to form an SDF-1/CXCR4 axis, which promotes proliferation and differentiation of neural precursor cells (NPCs). Moreover, it induces NPCs to migrate to damaged areas and adhere to positioning (13). However, during the occurrence of the in-origin nerve, only a small number of NPCs were able to mature and differentiate into neurons, successfully establishing synaptic connections in the pathological environment after ischemia with the limited repair of nerve function deficiencies (14).

The occlusion of the cerebral artery (middle cerebral artery occlusion, MCAO) demonstrated that SDF-1 can strengthen the connection between the surviving GABA neurons and the newborn neurons in the rat brain (15). It signifies that SDF-1 is likely to participate in the process of tissue integration around newborn neurons and local brain ischemia lesions, helping to restore the original structure and function.

In addition to the promotion of nerve regeneration after cerebral ischemia, SDF-1/CXCR4 illustrated that elevated levels of SDF-1 could promote vascular regeneration in the area around ischemic lesions $(16,17)$, with a correlation between nerve repair and blood vessels after the death of the cerebral infarction. The supportive and protective effect of blood vessels is the basis of nerve occurrence and nerve protection. Angiogenesis can provide a rich blood supply for nerve tissue, creating favorable conditions for the good construction of the microenvironment of nerve regeneration and further promotes the recovery of nerve function. Mao et al. assessed the involvement of endogenous endothelial progenitor cells (EPCs) in the formation of new blood vessels after stroke. They found an increase in SDF-1 expression in the ischemic boundary area after cerebral infarction, indicating that endogenous EPCs are involved in the formation of new blood vessels by CXCR4 / SDF-1 axis (18).

In summary, SDF-1 and CXCR4 are widely expressed in a variety of cells and tissues, performing a crucial role in the development of multiple sets of human systems. Moreover, they are closely related to inflammatory responses, angiogenesis, and the migration of neural stem cells (19). There is a wealth of research on SDF-1alpha/CXCR4 axes; nonetheless, it is undeniable that numerous applications of SDF-1 in other clinical areas have not been explored, and more in vitro experiments are needed in order to facilitate recovery after stroke.

The results of the current study pointed out that the ABCD2 scoring risk fraction was positively correlated with serum SDF-1, and the occurrence group had a significantly higher SDF-1 level, compared to the non-occurring group, with statistically significant differences. An increase in SDF-1 levels suggested a poor short-term (within 7 days) prognosis in TIA patients and could be used as a new biological indicator.

\section{Footnotes}

Conflicts of Interest: The study was supported by National Students' innovation and entrepre-neurship, China (201910367020) and Bengbu Science and Technology Innovation Program, Bengbu (20180335).

\section{References}

1. Simmatis LER, Scott SH, Jin AY. The impact of transient ischemic attack (TIA) on brain and behavior. Front Behav Neurosci. 2019;13:44. doi: 10.3389/fnbeh.2019.00044. [PubMed: 30914931].

2. Amarenco P, Lavallée PC, Labreuche J, Albers GW, Bornstein $\mathrm{NM}$, Canhão $\mathrm{P}$, et al. One-year risk of stroke after transient ischemic attack or minor stroke. $N$ Engl J Med. 2016; 374(16):1533-42. doi: 10.1056/NEJMoa1412981. [PubMed: 27096581].

3. Johnston SC, Rothwell PM, Nguyen-Huynh MN, Giles MF, Elkins JS, Bernstein AL, et al. Validation and refinement of scores to predict very early stroke risk after transient ischemic attack. Lancet. 2007;369(9558):283-92. doi: 10.1016/S01406736(07)60150-0. [PubMed: 17258668].

4. Xi HY, Si ZH, Li JC, Zhu JG, Yan HY. Assessment of cerebral infarction after transient cerebral ischemic attack by ABCD2 
score combined with the position of intracranial vascular stenosis. Medicine (Baltimore). 2019;98(15):e15081. doi: 10.1097/MD.0000000000015081. [PubMed: 30985660].

5. Zhao W, Zhao L, Liao J, Luo Y, He L. Early risk assessment of circulating endothelial progenitor cells and plasma stromal cell-derived factor-1 for nondisabling ischemic cerebrovascular events. BMC Neurol. 2019;19(1):22. doi: 10.1186/s12883-019-1250-5. [PubMed: 30755169].

6. The Chinese Society for Neurosurgery. Key points for diagnosis of various cerebrovascular diseases. Chin J Neurol. 1996; 29(6):379-80.

7. Kelly PJ, Albers GW, Chatzikonstantinou A, De Marchis GM, Ferrari J, George P, et al. Validation and comparison of imaging-based scores for prediction of early stroke risk after transient ischaemic attack: a pooled analysis of individualpatient data from cohort studies. Lancet Neurol. 2016; 15(12):1238-47. doi: 10.1016/S1474-4422(16)30236-8. [PubMed: 27751555].

8. Huan Y, Chaoyang Z, Kai D, Chunhua S, Xin Z, Yue Z. Predictive value of head-neck CTA combined with ABCD2 scale score for patients with cerebral infarction of vertebrobasilar transient ischemic attack (TIA). Med Sci Monit. 2018;24:9001-6. doi: 10.12659/MSM.909470. [PubMed: 30540723].

9. Ingram $\mathrm{N}$, Porter $\mathrm{CD}$. Transcriptional targeting of acute hypoxia in the tumour stroma is a novel and viable strategy for cancer gene therapy. Gene Ther. 2005;12(13):1058-69. doi: 10.1038/sj.gt.3302504. [PubMed: 15800661].

10. Zhou Z, Ren X, Zhou W, Zheng L. Willed-movement training reduces middle cerebral artery occlusion-induced motor deficits and improves angiogenesis and survival of cerebral endothelial cells via upregulating hypoxia-inducible factor- $1 \alpha$. Mol Med Rep. 2019;20(4):3910-6. doi: 10.3892/mmr. 2019.10578. [PubMed: 31432135].

11. Mollica Poeta V, Massara M, Capucetti A, Bonecchi R. Chemokines and chemokine receptors: new targets for cancer immunotherapy. Front Immunol. 2019;10:379. doi: 10.3389/fimmu.2019.00379. [PubMed: 30894861].
12. Legler DF, Thelen M. Chemokines: chemistry, biochemistry and biological function. Chimia (Aarau). 2016;70(12):856-9. doi: 10.2533/chimia.2016.856. [PubMed: 28661356].

13. Thored P, Arvidsson A, Cacci E, Ahlenius H, Kallur T, Darsalia V, et al. Persistent production of neurons from adult brain stem cells during recovery after stroke. Stem Cells. 2006;24(3):73947. doi: 10.1634/stemcells.2005-0281. [PubMed: 16210404].

14. Malone CD, Hasan SM, Roome RB, Xiong J, Furlong $M$, Opferman JT, et al. Mcl-1 regulates the survival of adult neural precursor cells. Mol Cell Neurosci. 2012;49(4):439-47. doi: 10.1016/j.mcn.2012.02.003. [PubMed: 22357134].

15. Ardelt AA, Bhattacharyya BJ, Belmadani A, Ren D, Miller RJ. Stromal derived growth factor-1 (CXCL12) modulates synaptic transmission to immature neurons during post-ischemic cerebral repair. Exp Neurol. 2013;248:246-53. doi: 10.1016/j.expneurol.2013.06.017. [PubMed: 23811016].

16. Li Y, Huang J, He X, Tang YH, Liu Y, Lin X, et al. Postacute stromal cell-derived factor- $1 \alpha$ expression promotes neurovascular recovery in ischemic mice. Stroke. 2014; 45(6):1822-9. doi: 10.1161/STROKEAHA.114.005078. [PubMed: 24876262].

17. Lan TH, Xu DP, Huang MT, Song JX, Wu HL, Li M. Ginsenoside $\mathrm{Rb} 1$ prevents homocysteine-induced EPC dysfunction via VEGF/p38MAPK and SDF-1/CXCR4 activation. Sci Rep. 2017;7(1):13061. doi: 10.1038/s41598-017-13436-7. [PubMed: 29026158].

18. Mao L, Huang M, Chen SC, Li YN, Xia YP, He QW, et al. Endogenous endothelial progenitor cells participate in neovascularization via CXCR4/SDF-1 axis and improve outcome after stroke. CNS Neurosci Ther. 2014;20(5):460-8. doi: 10.1111/cns.12238. [PubMed: 24581269].

19. Huang X, Wan M, Yang Q, Ding X, Zhou Z. The stromal cellderived factor-1 $\alpha(\mathrm{SDF}-1 \alpha) /$ cysteine-X-cysteine chemokine receptor 4 (CXCR4) axis: a possible prognostic indicator of acute ischemic stroke. J Int Med Res. 2019;47(5):1897-907. doi: 10.1177/0300060519827173. [PubMed: 30760134]. 